\title{
Laser-to-Fiber Coupling Scheme by Utilizing a Lensed Fiber Integrated with a Long-Period Fiber Grating
}

\author{
W. T. Chen and Lon A. Wang
}

\begin{abstract}
An optical coupling scheme between a laser diode and a single-mode fiber utilizing a lensed fiber integrated with a longperiod fiber grating is experimentally demonstrated and qualitatively analyzed. A long working distance of $110 \mu \mathrm{m}$ and a coupling efficiency of $35 \%$ are obtained for a laser diode with an ellipticity of 2.5. The longitudinal and transverse tolerances at 1-dB excess loss are 26 and $2.5 \mu \mathrm{m}$, respectively.
\end{abstract}

Index Terms-Long-period fiber gratings, optical coupling.

\section{INTRODUCTION}

$\mathbf{T}$ HE use of lensed fiber for coupling between a laser diode (LD) and a single-mode fiber (SMF) has been shown to have many desirable features such as being compact, stable, highly efficient, economical, and capable of extending to array-to-array coupling. However, several disadvantages of using lensed fiber can also be found: for example, small working distance (generally shorter than $10 \mu \mathrm{m}$ ), tight transverse tolerance $(\sim 0.3 \mu \mathrm{m}$ for $1-\mathrm{dB}$ loss $)$, and critical fabrication process (lens radius $\sim 5 \mu \mathrm{m}$ ) [1], [2]. In practice, a working distance longer than $\sim 100 \mu \mathrm{m}$ is desired for the automatic packaging process of an optical subassembly (OSA) module, and for the reduction of optical backreflection. For example, the estimated optical backreflections are $\sim-30$ and $-60 \mathrm{~dB}$ at working distances of 10 and $100 \mu \mathrm{m}$, respectively, for the lensed fiber with radii of $\sim 50 \mu \mathrm{m}$ [3]. Although an integrated spot size converter has been reported for improving coupling [4], the working distance is typically within $20 \mu \mathrm{m}$, and optical backreflection may impose an issue for an uncoated cleave fiber. Many other conventional approaches have been developed to increase the working distance; however, the distance is still less than $40 \mu \mathrm{m}$ due to the limitation resulted from the small core diameter of a SMF [2], [5].

Although another lensed fiber coupling scheme utilizing a graded-index fiber and a hemispherical coreless fiber could obtain a larger working distance than the conventional ones, the fabrication processes are relatively complicated since three different types of fibers are used for integration [6]. To overcome the above disadvantages, we have previously proposed an alternative coupling scheme between two fibers by integrating an LPFG with a hemispherical lens at the fiber end [7]. Here

Manuscript received January 12, 2000.

The authors are with the Department of Electrical Engineering and Institute of Electro-Optical Engineering, National Taiwan University, Taipei, Taiwan 106, R.O.C. (e-mail: lon@ccms.ntu.edu.tw).

Publisher Item Identifier S 1041-1135(00)03660-0.

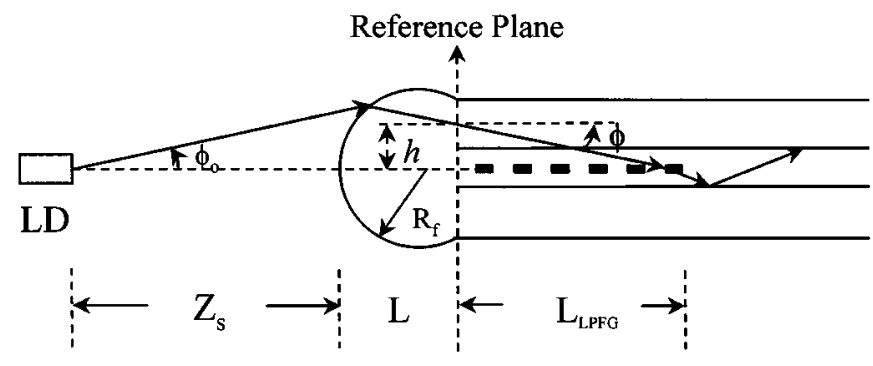

Fig. 1. Schematic diagram of the proposed coupling scheme.

we demonstrate that such a scheme can also be applied to coupling light from an LD with an ellipticity of 2.5 to an SMF at a working distance larger than $100 \mu \mathrm{m}$.

\section{PRINCIPLE}

The proposed new coupling scheme is schematically shown in Fig. 1, in which ray optics is used for qualitative analysis. Rays emitting from an LD at an output angle $\phi_{o}$ are coupled into the cladding of a receiving SMF by a hemispherical fiber lens. The rays that pass through the LPFG will be diffracted into different orders. A portion of rays satisfying the following condition at the reference plane will be coupled into the SMF and remain propagated in the core:

$$
\begin{aligned}
a_{\mathrm{cl}}>|h| & >a_{\mathrm{co}} \\
\alpha<|\phi| & <\frac{\pi}{2}-\sin ^{-1}\left(1-\frac{m \lambda}{n_{\mathrm{cl}} \Lambda}\right)
\end{aligned}
$$

where $\phi$ and $h$ are the input angle and height; $\Lambda$ and $L_{\mathrm{LPFG}}$ are the period and length of LPFG; $a_{\mathrm{co}}$ and $a_{\mathrm{cl}}$ are the radii of core and cladding, respectively; $m$ is the order of diffraction; $n_{\mathrm{cl}}$ is the refractive index of cladding; and parameter $\alpha$ is a function of $L_{\mathrm{LPG}}$ and $h$. Other rays accepted by the core may be coupled into the cladding due to the diffraction of LPFG. As shown in Fig. 2, there are four acceptable regions (solid boxes) when a lensed LPFG is used and one region without the lensed LPFG (dashed box). We show the coupling characteristics at various working distance $Z_{s}$ for a lens, for example, having a radius of curvature of $50 \mu \mathrm{m}$. The following parameters are assumed: $\lambda=1.55 \mu \mathrm{m}, \Lambda=400 \mu \mathrm{m}, n_{c o}=1.458, n_{c l}=1.45$, $a_{\mathrm{co}}=4.5 \mu \mathrm{m}, a_{\mathrm{cl}}=62.5 \mu \mathrm{m}, L=25 \mu \mathrm{m}$, and $L_{\mathrm{LPFG}}=25$ $\mathrm{mm}$. Each curve is obtained by ray tracing at an increment of one degree output angle $\phi_{o}$. The results show that within a long working distance, i.e., from 60 to $160 \mu \mathrm{m}$ for the current example, most rays are accepted by the cladding and are diffracted 


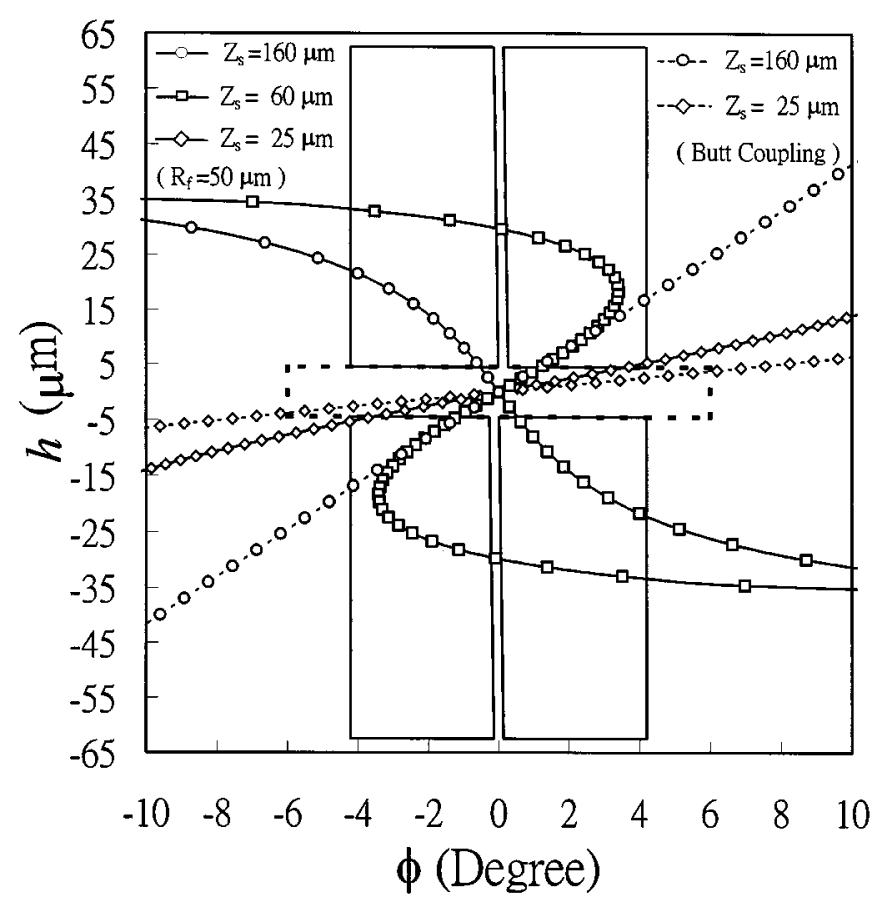

Fig. 2. Simulated results of acceptable region for coupling with LPFG (four solid boxes) and without LPFG (the dashed box). Input angle $\phi$ versus input height $h$ for various working distances are plotted for every one degree of the output angle $\phi_{o}$ (open label).

into the core owing to the assistance of LPFG. In contrast, for a short working distance of about 5-25 $\mu \mathrm{m}$, a large amount of rays will be diffracted into cladding because they fall in the core region.

\section{EXPERIMENT RESULTS}

An LPFG is imprinted in a dispersion-shifted SMF by using 248-nm exposure wavelength through an amplitude mask of period $380 \mu \mathrm{m}$, in which the SMF has been prehydrogenated for 15 days at a pressure of 1500 psi. In order to obtain a larger bandwidth of LPFG, the ultraviolet (UV)-exposed length is controlled to be $15 \mathrm{~mm}$. An additional post-dc exposure is employed to tune the resonant wavelength of $\mathrm{HE}_{11}$ mode of LPFG to match the peak wavelength of the laser diode after annealing. The SMF is cleaved near the endface of the UV-exposed area and then an electrical arc discharge is utilized to form a hemispherical lens. The radius of hemispherical lens is controlled by discharge voltage. However, when the desired radius is less than $70 \mu \mathrm{m}$, a chemical etching may be preferred.

A Fabry-Perot (FP)-type LD operating at a wavelength of $1.56 \mu \mathrm{m}$ is used. The divergence angle, defined at full width of half maximum, is measured as 15 and 38 degrees for the parallel and perpendicular directions, respectively. The corresponding beam waists, defined at $1 / e^{2}$ of the maximum intensity, are estimated to be 2.1 and $0.9 \mu \mathrm{m}$, respectively. The fabricated lensed fiber of LPFG and FP-LD are placed on the holders of an alignment system equipped with a charge-coupled-device camera. It is noted that the resonant peak of LPFG is well tuned to match the peak wavelength of FP-LD. The bandwidth of LPFG

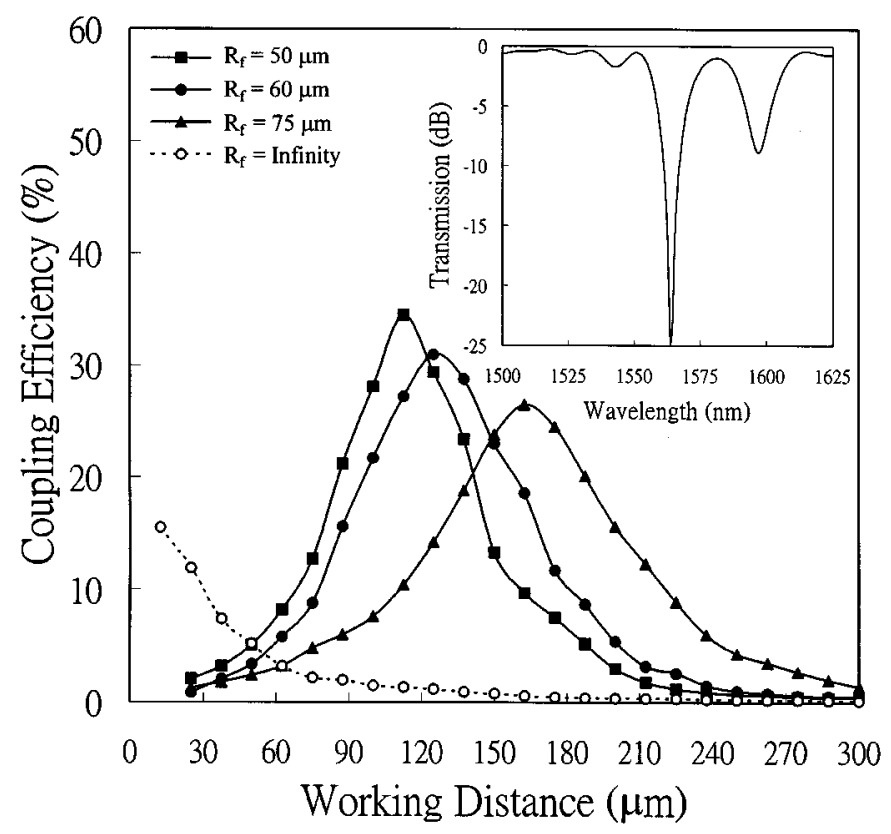

Fig. 3. Coupling efficiency versus working distance for $\mathrm{HE}_{11}$ mode by using lensed fibers with various radii. The inset shows the transmission spectrum of the LPFG being used.

( $\sim 15 \mathrm{~nm}$, defined at $3 \mathrm{~dB}$ ) is controlled to be larger than the LD's ( $\sim 4 \mathrm{~nm}$, defined at $10 \mathrm{~dB})$.

Fig. 3 depicts the coupling efficiency versus working distance for various radii. The solid and dashed curves correspond to the case of butt coupling (i.e., infinite $R_{f}$ and without LPFG) and lensed fibers integrated with LPFG's, respectively. The inset shows the transmission spectrum of used LPFG, in which a transmission loss of $\sim 25 \mathrm{~dB}$ is obtained for the first cladding mode. For the case of butt coupling, the coupling efficiency reaches the maximum of $\sim 15.5 \%$ at a very short working distance of $\sim 12 \mu \mathrm{m}$, and then decreases monotonically with separation. Conversely, for lensed fibers integrated with LPFG, only very small coupling efficiencies are measured at short working distances, but the efficiencies increase to maxima at markedly long working distances. These results are consistent qualitatively with those shown in Fig. 2. It is seen that for the LPFG with 50- $\mu \mathrm{m}$-radius lens, the rays having the output angles $\phi_{o}$ from $+2^{\circ}$ to $+8^{\circ}$ and $-2^{\circ}$ to $-8^{\circ}$ can be accepted by the LPFG at the working distance of $160 \mu \mathrm{m}$, but no rays are acceptable by the LPFG for working distance less than $25 \mu \mathrm{m}$. In contrast, in the butt-coupling case, the rays having the output angles $\phi_{o}$ from $-9^{\circ}$ to $\sim+9^{\circ}$ can be accepted by the SMF for working distance within $25 \mu \mathrm{m}$, but only a few rays are accepted by the SMF at a working distance of $160 \mu \mathrm{m}$. For example, over a working distance of $\sim 60$ to $160 \mu \mathrm{m}$, the measured coupling efficiency for the LPFG with $50-\mu$ m-radius lens is $2.5-16$ times larger than that of butt coupling. For a lens radius of $50 \mu \mathrm{m}$, the maximum measured coupling efficiency is about $34.5 \%$ at a working distance of $110 \mu \mathrm{m}$, and the corresponding longitudinal and transverse tolerances are about 26 and $2.6 \mu \mathrm{m}$ at 1-dB loss. For other radii of 60 and $75 \mu \mathrm{m}$, the optimal working distances are 125 and $160 \mu \mathrm{m}$ with corresponding coupling efficiencies of 31 and $26 \%$, respectively. Their longitudinal tolerances are about 40 and $50 \mu \mathrm{m}$, and the transverse tolerances are 2.8 and $3.2 \mu \mathrm{m}$ 


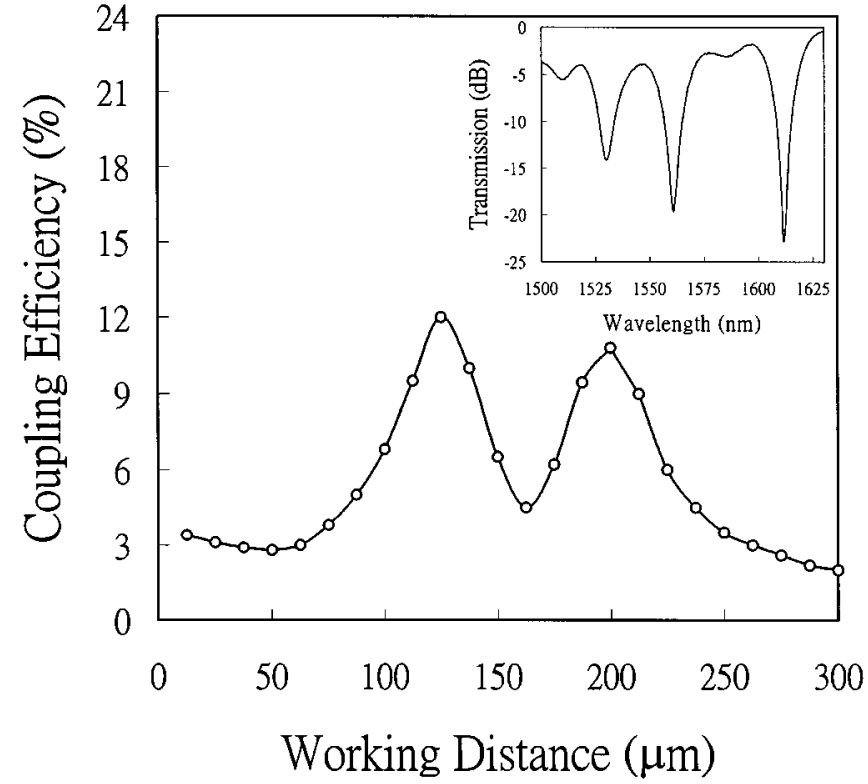

Fig. 4. Coupling efficiency versus working distance for $\mathrm{HE}_{13}$ mode. The inset shows the transmission spectrum of the LPFG being used.

at $1-\mathrm{dB}$ loss for fibers with radii of 60 and $75 \mu \mathrm{m}$, respectively. For comparison, the measured longitudinal and transverse tolerances of butt-coupling are about 10 and $2 \mu \mathrm{m}$.

To study the effect of mode dependence, another LPFG whose transmission loss is $10 \mathrm{~dB}$ in mode $\mathrm{HE}_{11}$ and $15 \mathrm{~dB}$ in mode $\mathrm{HE}_{13}$ is used. The resonant peak of cladding mode $\mathrm{HE}_{13}$ is tuned to match the mean wavelength of FP-LD. The radius of curvature of the lensed fiber $R_{f}$ is about $75 \mu \mathrm{m}$. The coupling efficiency versus working distance is shown in Fig. 4, and the inset depicts the transmission spectrum of used LPFG. As contrasted with $\mathrm{HE}_{11}$ for $R_{f}=75 \mu \mathrm{m}$, the coupling characteristics of $\mathrm{HE}_{13}$ mode have the following features.

1) There are two maxima of coupling efficiency at the working distances of 120 and $180 \mu \mathrm{m}$.

2) The maximum coupling efficiency is only about half of $\mathrm{HE}_{11}$.
3) The location of the maximum coupling efficiency of $\mathrm{HE}_{11}$ is nearly at a local minimum of $\mathrm{HE}_{13}\left(Z_{s} \sim 160 \mu \mathrm{m}\right)$.

Therefore, the results show that the coupling efficiency is strongly cladding-mode dependent. Other dependence of coupling efficiency such as magnitude of transmission loss of an LPFG, wavelength difference between laser and LPFG, and the fiber characteristics will be reported elsewhere.

\section{CONCLUSION}

We have demonstrated a long-working-distance coupling scheme between an FP-LD and a hemispherical lensed fiber integrated with an LPFG. With an appropriate design on radius and control on matching the resonant peak of an LPFG, coupling efficiencies of more than $30 \%$ and working distances more than $120 \mu \mathrm{m}$ can be obtained for cladding mode $\mathrm{HE}_{11}$. Such a coupling scheme may be helpful in the packaging process of an OSA module owing to its long working distance.

\section{REFERENCES}

[1] J. Yamada, Y. Murakami, J. Sakai, and T. Kimura, "Characteristics of a hemispherical microlens for coupling between a semiconductor laser and single-mode fiber," IEEE J. Quantum. Electron., vol. QE-16, pp. $1067-1072,1980$

[2] N. Kalonji and J. Semo, "High efficiency long working distance laser diode to singlemode fiber coupling arrangement," Electron. Lett., vol. 30, pp. 892-893, 1994.

[3] W. Bludau and R. H. Rosserg, "Low-loss laser-to-fiber coupling with negligible optical feeback," J. Lightwave Technol., vol. LT-3, pp. 294-302, 1985.

[4] I. Moerman, P. P. Van Daele, and P. M. Demeester, "A review on fabrication technologies for the monolithic integration of tapers with III-V semiconductor devices," IEEE J. Select. Topics Quantum Electron., vol. 3, pp. 1308-1320, 1997.

[5] H. Kuwahara, M. Sasaki, and N. Tokoyo, "Efficient coupling from semiconductor lasers into single-mode fibers with tapered hemispherical ends," Appl. Opt., vol. 19, pp. 2578-2583, 1980.

[6] K. Shiraishi, H. Ohnuki, N. Hiraguri, K. Matsumura, I. Ohishi, H. Morichi, and H. Kazami, "A lensed-fiber coupling scheme utilizing a graded-index fiber and a hemispherically ended coreless fiber tip," $J$. Lightwave Technol., vol. 15, pp. 356-362, 1997.

[7] W. T. Chen and L. A. Wang, "Optical coupling between singlemode fibers by utilising long-period fiber gratings," Electron. Lett., vol. 35, pp. 421-423, 1999. 